\title{
The adjuvant regimen for resected EGFR mutated patients: the "same-for-all-therapy" or not?
}

\author{
Cesare Gridelli, Francesca Casaluce \\ Division of Medical Oncology, A.O.R.N. San Giuseppe Moscati, Avellino, AV, Italy \\ Correspondence to: Cesare Gridelli. Division of Medical Oncology, A.O.R.N. S.G. Moscati Hospital, Contrada Amoretta, 83100, Avellino, Italy. \\ Email: cgridelli@libero.it. \\ Comment on: Liang W, Cai K, Chen C, et al. Society for Translational Medicine consensus on postoperative management of EGFR-mutant lung \\ cancer (2019 edition). Transl Lung Cancer Res 2019;8:1163-73.
}

Submitted Apr 06, 2020. Accepted for publication Apr 17, 2020.

doi: $10.21037 /$ atm-20-3170

View this article at: http://dx.doi.org/10.21037/atm-20-3170

If metastatic non-small cell lung cancer (NSCLC) treatment has clearly moved away from the "same-for-all-therapy" towards precision therapy strategy, in the adjuvant setting the choice of treatment is not guided by molecular analyses, with cisplatin-based doublet chemotherapy as standard of care in stages II and III disease. However, a significant fall in overall survival (OS) was observed for stage IB-IIIA, as well as high rates of recurrence and/or metastasis inevitably still occurred. Improving current adjuvant strategy, new investigational approaches are under evaluation, including targeted therapy. The impressive superiority of an Epidermal Growth Factor Receptor (EGFR) tyrosine kinase inhibitor (TKI) targeted therapy than chemotherapy as upfront choice for advanced EGFR mutated NSCLC patients laid the rational of several adjuvant clinical trials, evaluating potential benefit of this "tailored choice" in earlier stage disease also. This Chinese expert consensus would provide recommendations on key issues, focusing on current evidence and suggesting future implications for clinical practice. Among Chinese experts, EGFR mutation profiling is strongly routinely recommended in surgically resected non-squamous NSCLC. When EGFRsensitizing mutations occurs, patients might be suitable of three main adjuvant options: standard chemotherapy, an EGFR-TKI at least for 2 years, and sequential use of both modes of therapy. Stratifying recurrence risk, the adjuvant EGFR-TKI choice could be mainly used in those patients with stage II-IIIA EGFR-mutated NSCLC, at high risk of recurrence and less tolerant to chemotherapy. At recurrence, the use of EGFR-TKI (preferably osimertinib) as salvage therapy is strongly recommended in all resected EGFR-mutant patients, also in those previously receiving an adjuvant EGFR-TKI drug, but only if relapsed after drug discontinuation (1).

Clearly, several controversial questions were involved in this consensus, opening issues to future debate. Based on literature data before IPASS trial publication, two trialsthe JBR19 and the RADIANT_-firstly compared two years of adjuvant gefitinib and erlotinib to placebo, respectively, but randomizing EGFR unselected population after completion of any planned adjuvant chemotherapy in stage IB-IIIA NSCLC $(2,3)$. Not surprising, both trials were clearly negative, failing to significantly prolong disease free survival (DFS) also in 161 EGFR-mutated patients included in erlotinib arm of RADIANT trial (HR: 0.61), mainly due to the hierarchical design of this study. Extrapolating knowledge from advanced stage trials and focusing exclusively on EGFR-mutant patients with the highest risk of recurrence (N1-N2), the ADJUVANT and the EVAN studies compared directly the adjuvant TKIs—gefitinib for two years and erlotinib for one year, respectively-with standard chemotherapy $(4,5)$. Both trials met their primary endpoint, delaying significantly the time of progression/ recurrence of disease with decreased toxicities, but still not providing enough data to support a worldwide conclusion, above all for lacking OS data and for their accrual in a Chinese population.

A systematic review and meta-analysis of six adjuvant EGFR-TKIs trials, including a total of 1,860 resected patients, was recently published, confirming risk of disease 
recurrence decreased by $48 \%$ in patients harboring an EGFR mutation, with higher DFS benefit in trials using TKIs over chemotherapy compared with trials using TKIs postchemotherapy. However, OS improvement were not reported (HR: 0.64), remaining still uncertain this key-point (6). Other two recent meta-analyses reported similar results, showing that EGFR-TKI-based therapy was associated with better DFS compared with non-EGFR-TKI-based adjuvant therapy in patients with NSCLC harboring EGFR mutation $(7,8)$. As presented at American Society of Clinical Oncology (ASCO) 2019, the Chinese meta-analysis of 11 trials (including a total of 1,152 resected NSCLC patients with activating EGFR mutations) reported that adjuvant treatment with EGFR-TKIs prolonged both OS and DFS when compared to treatment without adjuvant TKIs. From predefined subgroup analyses, adjuvant EGFR-TKI prolonged significantly DFS rather than chemotherapy, but without OS differences. Notably, the combination of an EGFR-TKI to chemotherapy was associated with significantly longer DFS as well as OS than chemotherapy alone, suggesting a new potential option (8).

Clearly, data from literature are too weak for strongly recommend an EGFR-TKI therapy as adjuvant choice. Focusing on two recent trials, EVAN was a small size study enrolling only patients with stage IIIA disease, while promising data from the larger ADJUVANT trial have raised several questions. Given the preponderance of stage III patients in ADJUVANT trial, and lack of benefit in N1 patients in the forest plot, it is not clear how this will translate to patients diagnosed at earlier stages. Another issue is the beneficial variety emerged from the study, where $40 \%$ patients experienced relapse within two years of gefitinib treatment. With aim to identify patients precisely using other predictive biomarkers beyond EGFR, a comprehensive tumor genomic analyses on ADJUVANT patients was recently performed, reporting the predictive MEDUSA model, integrating from five predictive biomarkers (TP53, NKX2-1, CDK4, MYC \& RB1), with RB1 mutation or copy number loss as the only predictor in favor of chemotherapy (9). Also, alterations of the tumor microenvironment (TME) might help to identifying individuals who will benefit from adjuvant EGFR-TKI therapy, as recently reported. According to eight key TME genes, patients were clustered in two groups with distinct immune profiles, with aim to identify individuals with "inflamed" phenotype (higher lymphocyte infiltration score and TGF- $\beta$ response), resulting with both DFS and diseasespecific survival significantly prolonged (10).
Whether adjuvant EGFR-TKIs are able to improve cure rates or whether they just delay recurrence is the second unanswered key question to solve, considering OS as the main primary endpoint for most clinical trials. Actually, considering the timeframe for accrual and the longer follow-up needed for a definitive assessment of OS benefit, DFS might be a suitable surrogate endpoint, but clearly not still sufficient to change the global clinical practice. Interestingly, patients who recurred after adjuvant erlotinib or gefitinib still have the opportunity to be re-challenged with other TKIs, whose median duration of treatment approximated the PFS in a de novo advanced EGFR-mutant population $(11,12)$. However, the use of third generation TKI osimertinib should be "the preferable" but not "the generalized" choice for patients who relapsed, considering data of FLAURA trial enrolling mostly patients who presented with stage IV disease de novo.

Other two main issues under debate are the optimal EGFR-TKI drug to be used with the best tolerability and efficacy management, as well as the optimal duration of treatment. Clearly, longer treatment may be a burden to patients and could lead to decreased treatment compliance as well as cumulative toxicities. Notably, about $80 \%$ of patients of ADJUVANT trial received gefitinib for one year, with $68 \%$ prolonging therapy to more than 18 months. In SELECT trial, about $70 \%$ of patients completed at least 22 of preplanned 24 months of erlotinib therapy, but reducing dose to $100 \mathrm{mg} /$ die in $40 \%$ patients (16\% needed second dose reduction to $50 \mathrm{mg} / \mathrm{die}$ ). Focusing on EGFRTKIs beyond first-generation, data from phase II trial was presented at ASCO 2019, comparing two different length of afatinib treatment ( 3 months $v s .2$ years) after completion of standard adjuvant therapy (13). As expected, the longlasting afatinib arm payed the price of higher toxicities with subsequent more discontinuation ( $18 \%$ vs. $4 \%$ for $2-y r$ and 3 -months arms, respectively) and lower compliance, with only $50 \%$ of patients completing the preplanned two year of treatment rather than $92 \%$ of three moths choice. Consistently with prior EGFR-TKIs adjuvant trials, many patients in the 2-year arm did not complete treatment, with withdrawal of consent being the main reason $(23 \%)$, followed by toxicity $(18 \%)$ and recurrence $(9 \%)$. So, in the era of TKIs with improved tolerance, the optimum length of therapy remains an important unsolved question. Analyzing data from ADJUVANT trail, planned gefitinib for 24 months delayed significantly recurrences of 10.7 months rather than conventional chemotherapy (median DFS: $28.7 v$ s. 18.0 months), but the duration of benefit after the 
Table 1 Ongoing phase III adjuvant EGFR TKIs trial

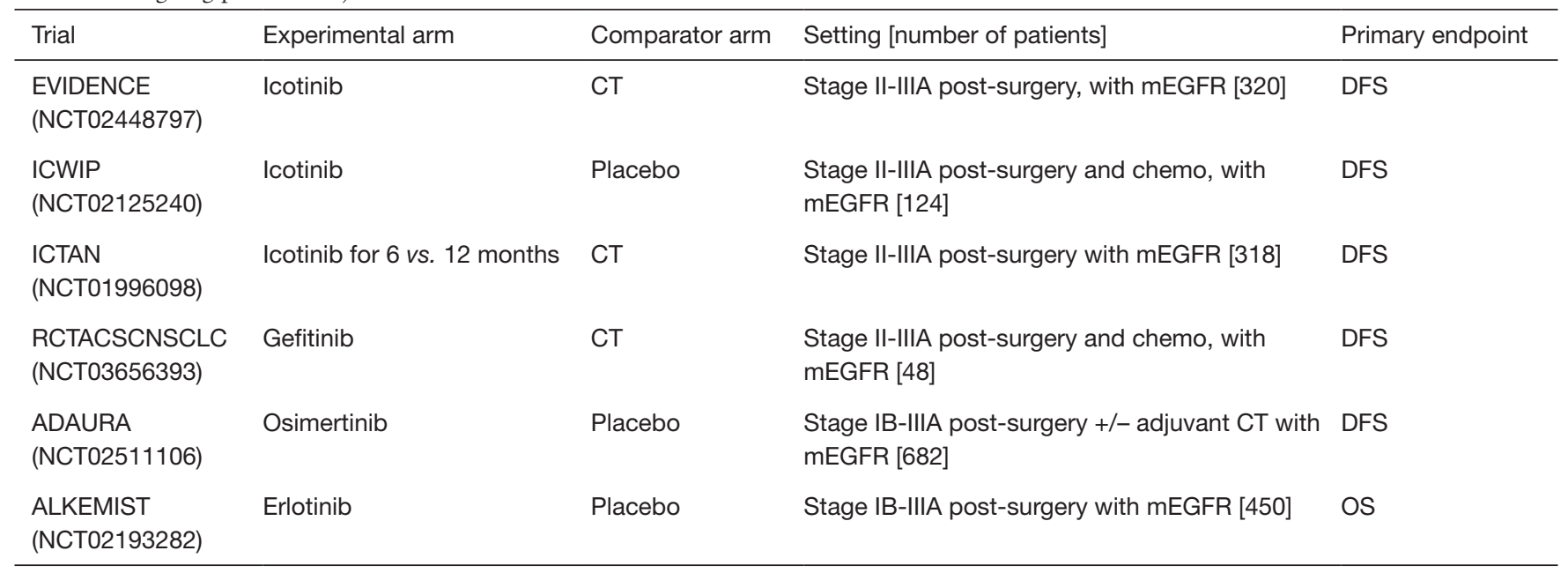

CT, chemotherapy; DFS, disease free survival; mEGFR, mutated EGFR; OS, overall survival.

discontinuation of TKI might be limited, as suggested by collide DFS curves at 36 months, with "no progressor tail". On other hand, the 2-year afatinib regimen induced a $14 \%$ reduction of recurrence rates compared to the 3-month schedule (DFS at 2 years: $85 \%$ vs. 71\%). Although this difference was not statistically different and considering that the trial was early closed at 46 of 60 planned patients for slow accrual, this delayed recurrence taste similarly to those reported with 2-year of erlotinib in SELECT trial (DFS 2-yr: $88 \%$ ), suggesting that administering an EGFR-TKI after standard adjuvant chemo/radiotherapy could provide better long term outcome than TKI therapy alone (DFS 2-yr: $57 \%$ in gefitinib arm of ADJUVANT trial).

About detection of EGFR mutation profiling in surgically resected NSCLC, data from literature suggested the potential rational for this earlier stage screening, as ADAURA trial, where high prevalence of EGFR mutations associated with EGFR-TKI sensitivity (ex19del, L858R) were reported in Asian and female patients with resected stage IB-IIIA NSCLC, consistently with the advanced setting. However, this detection should be still optional and not routinely recommended, considering the unclear demonstration of targeted therapy utility and the minimal data of EGFR mutant positivity in non-smoking early resectable squamous NSCLC (14).

Currently, new phase III trials testing are ongoing (Table 1), evaluating for adjuvant EGFR-TKIs beyond first- and second-generation agents, comparing shorter versus longer treatment durations and focusing also on new potential biomarkers to help selection of patients-efforts.
Their results will more clearly establish the role, if any, for adjuvant TKI therapy. At the present time, the choice of an adjuvant therapy should not be guided by molecular analyses and targeted therapy should not be used in the adjuvant setting. The ideal treatment might have no side effects with a defined survival benefit. On other hand, if the drug might not doing more that postponing the relapse-if and where happen - most people could be subjected to costs/toxicities of an useless choice. Further research is mandatory to determine also how best to integrate treatment (e.g., chemotherapy, radiotherapy, and immunotherapy) into the adjuvant strategy, and results are strongly awaited.

\section{Acknowledgments}

Funding: None.

\section{Footnote}

Provenance and Peer Review: This article was commissioned by the editorial office, Annals of Translational Medicine. The article did not undergo external peer review.

Conflicts of Interest: Both authors have completed the ICMJE uniform disclosure form (available at http://dx.doi. org/10.21037/atm-20-3170). CG, a speaker bureau and advisory board member, reports other from Astra Zeneca, other from BMS, other from MSD, other from Roche, outside the submitted work; FC has no conflicts of interest to declare. 
Ethical Statement: The authors are accountable for all aspects of the work in ensuring that questions related to the accuracy or integrity of any part of the work are appropriately investigated and resolved.

Open Access Statement: This is an Open Access article distributed in accordance with the Creative Commons Attribution-NonCommercial-NoDerivs 4.0 International License (CC BY-NC-ND 4.0), which permits the noncommercial replication and distribution of the article with the strict proviso that no changes or edits are made and the original work is properly cited (including links to both the formal publication through the relevant DOI and the license). See: https://creativecommons.org/licenses/by-nc-nd/4.0/.

\section{References}

1. Liang W, Cai K, Chen C, et al. Society for Translational Medicine consensus on postoperative management of EGFR-mutant lung cancer (2019 edition). Transl Lung Cancer Res 2019;8:1163-73.

2. Goss GD, O'Callaghan C, Lorimer I, et al. Gefitinib versus placebo in completely resected non-small-cell lung cancer: results of the NCIC CTG BR19 study. J Clin Oncol 2013;31:3320-6.

3. Kelly K, Altorki NK, Eberhardt WE, et al. Adjuvant Erlotinib Versus Placebo in Patients With Stage IBIIIA Non-Small-Cell Lung Cancer (RADIANT): A Randomized, Double-Blind, Phase III Trial. J Clin Oncol 2015;33:4007-14.

4. Zhong WZ, Wang Q, Mao WM, et al. Gefitinib versus vinorelbine plus cisplatin as adjuvant treatment for stage II-IIIA (N1-N2) EGFR-mutant NSCLC (ADJUVANT/ CTONG1104): a randomised, open-label, phase 3 study. Lancet Oncol 2018;19:139-48.

5. Yue D, Xu S, Wang Q, et al. Erlotinib versus vinorelbine plus cisplatin as adjuvant therapy in Chinese patients with stage IIIA EGFR mutation-positive non-small-cell lung cancer (EVAN): a randomised, open-label, phase 2 trial. Lancet Respir Med 2018;6:863-73.

Cite this article as: Gridelli C, Casaluce F. The adjuvant regimen for resected EGFR mutated patients: the "same-forall-therapy" or not? Ann Transl Med 2020;8(18):1195. doi: 10.21037/atm-20-3170
6. Raphael J, Vincent M, Boldt G, et al. Adjuvant Epidermal Growth Factor Receptor Tyrosine Kinase Inhibitors (TKIs) in Resected Non-Small Cell Lung Cancer (NSCLC), A Systematic Review and Meta-analysis. Am J Clin Oncol 2019;42:440-5.

7. Wu JX, He Q, Ye F, et al. EGFR-TKI-based vs nonEGFR-TKI-based adjuvant therapy in resected non-smallcell lung cancer with EGFR mutations: a meta-analysis of randomized controlled trials. Onco Targets Ther 2018;11:6803-10.

8. Xie P, Tang $\mathrm{W}, \mathrm{Li} \mathrm{X}$, et al. The role of EGFR inhibitors as adjuvant therapy for EGFR mutation positive non-small cell lung cancer. J Clin Oncol 2019;37:8508.

9. Wu YL, Liu SY, Wang Q, et al. A comprehensive model of genetic-features predicts outcome of personalized adjuvant treatment in resected EGFR-mutant stage IIIIIA NSCLC: Results from a phase III trial (CTONG 1104-ADJUVANT), Ann of Oncol 2019;30:v586.

10. Khalil M, Elkhanany A, Yang Y, et al. The tumor microenvironment in EGFR-driven loco-regional lung adenocarcinoma can predict higher risk of recurrence. J Clin Oncol 2019;37:8521.

11. Pennell NA, Neal JW, Chaft JE, et al. SELECT: A Phase II Trial of Adjuvant Erlotinib in Patients With Resected Epidermal Growth Factor Receptor-Mutant Non-SmallCell Lung Cancer. J Clin Oncol 2019;37:97-104.

12. Oxnard GR, Janjigian YY, Arcila ME, et al. Maintained sensitivity to EGFR tyrosine kinase inhibitors in EGFRmutant lung cancer recurring after adjuvant erlotinib or gefitinib. Clin Cancer Res 2011;17:6322-8.

13. Chaft JE, Costa DB, Muzikansky A, et al. Randomized phase II study of adjuvant afatinib for three months versus two years in patients with resected stage I-III EGFR mutant NSCLC. J Clin Oncol 2019;37:8507.

14. Tsuboi M, Herbst RS, John T, et al. Frequency of epidermal growth factor receptor (EGFR) mutations in stage IB-IIIA EGFR mutation positive non-small-cell lung cancer (NSCLC) after complete tumour resection. Ann of Oncol 2019;30:v585-90. 